\title{
Comparison of Categorical Color Perception in Two Estrildid Finches
}

\author{
Eleanor M. Caves, ${ }^{1,2, \star}$ Patrick A. Green, ${ }^{1,2}$ Matthew N. Zipple, ${ }^{2}$ Dhanya Bharath, ${ }^{3}$ Susan Peters, ${ }^{2}$ \\ Sönke Johnsen, ${ }^{2}$ and Stephen Nowicki ${ }^{2,4, *}$
}

1. Centre for Ecology and Conservation, College of Life and Environmental Sciences, University of Exeter, Penryn, Cornwall TR10 9FE, United Kingdom; 2. Department of Biology, Duke University, Durham, North Carolina 27708; 3. Indian Institute of Science, Bengaluru, Karnataka 560012, India; 4. Department of Neurobiology, Duke University Medical School, Durham, North Carolina 27708

Submitted June 2, 2020; Accepted September 17, 2020; Electronically published December 23, 2020

Online enhancements: supplemental figures and tables. Dryad data: https://doi.org/10.5061/dryad.stqjq2c1z.

ABSTRACT: Sensory systems are predicted to be adapted to the perception of important stimuli, such as signals used in communication. Prior work has shown that female zebra finches perceive the carotenoid-based orange-red coloration of male beaks - a mate choice signal - categorically. Specifically, females exhibited an increased ability to discriminate between colors from opposite sides of a perceptual category boundary than equally different colors from the same side of the boundary. The Bengalese finch, an estrildid finch related to the zebra finch, is black, brown, and white, lacking carotenoid coloration. To explore the relationship between categorical color perception and signal use, we tested Bengalese finches using the same orange-red continuum as in zebra finches, and we also tested how both species discriminated among colors differing systematically in hue and brightness. Unlike in zebra finches, we found no evidence of categorical perception of an orange-red continuum in Bengalese finches. Instead, we found that the combination of chromatic distance (hue difference) and Michelson contrast (difference in brightness) strongly correlated with color discrimination ability on all tested color pairs in Bengalese finches. The pattern was different in zebra finches: this strong correlation held when discriminating between colors from different categories but not when discriminating between colors from within the same category. These experiments suggest that categorical perception is not a universal feature of avian - or even estrildid finch - vision. Our findings also provide further insights into the mechanism underlying categorical perception and are consistent with the hypothesis that categorical perception is adapted for signal perception.

Keywords: zebra finch, Bengalese finch, color signaling, avian vision, carotenoid, sensory ecology.

\footnotetext{
*Corresponding authors; email: snowicki@duke.edu, eleanor.caves@gmail .com.
}

ORCIDs: Caves, https://orcid.org/0000-0003-3497-5925; Green, https:// orcid.org/0000-0002-2434-8795; Zipple, https://orcid.org/0000-0003-3451 -2103; Bharath, https://orcid.org/0000-0003-1138-8525; Johnsen, https:// orcid.org/0000-0002-3943-8320; Nowicki, https://orcid.org/0000-0002-6564 $-905 \mathrm{X}$

Am. Nat. 2021. Vol. 197, pp. 190-202. (C) 2020 by The University of Chicago. 0003-0147/2021/19702-60002\$15.00. All rights reserved.

DOI: $10.1086 / 712379$

\section{Introduction}

To efficiently and accurately assess signals, animals filter out irrelevant information or simplify or enhance relevant variation using a variety of perceptual mechanisms (Wehner 1987). One such mechanism is categorical perception, in which an animal's perceptual system sorts continuously varying stimuli into discrete categories (Harnad 1987). With categorical perception, individuals sort, or "label," continuous variation along some dimension of a stimulus into categories. Individuals also exhibit enhanced discrimination of stimuli from different sides of the category boundary compared with equally different stimuli from within the same category. Although the stimulus varies continuously, certain equally distinct variants are perceived as more distinct from one another depending on whether they are located on the same side or different sides of a category boundary. The extent to which categorical perception functions in signal assessment has important implications for our understanding of the selection pressures shaping both perceptual processes and signal form (Green et al. 2020).

Originally described for human speech (Liberman et al. 1957), categorical perception has since been demonstrated in signaling contexts across a variety of taxa and sensory modalities (reviewed in Green et al. 2020). Recently, female zebra finches (Taeniopygia guttata, family Estrildidae) were shown to categorically perceive an orange-red color range that corresponds to variation in the carotenoid-based coloration of male zebra finch beaks (Caves et al. 2018), a signal that females assess during mate choice (Burley and Coopersmith 1987; de Kogel and Prijs 1996; Blount et al. 2003). Female zebra finches also categorically perceive a blue-green color range that has no known signaling function in this species (Zipple et al. 2019), but key differences exist between the structure of blue-green and orange-red categories. In particular, females exhibit greater withincategory discrimination in the blue-green color range 
compared with the orange-red color range. One potential explanation for these differences is that selection has acted on how the visual system perceives orange-red coloration given that it plays an important signaling role. Comparative studies, particularly between species with similar visual physiologies but different signaling traits, provide a powerful tool by which to examine the selective influence of signal perception on perceptual processing and how receivers may coevolve with signalers (Price 2017).

Here, we test for categorical perception of carotenoidbased coloration in another estrildid finch, the Bengalese finch (Lonchura striata domestica). Domesticated primarily from the white-rumped munia (Lonchura striata) within the past 250 years (Colquitt et al. 2018), Bengalese finches display characteristic piebald coloration, with birds ranging from nearly all white to nearly all black (Eisner 1960). Bengalese finches and their wild ancestor are sexually monomorphic and aside from black, brown, and white display no obvious coloration, including carotenoid-based coloration. This lack of a carotenoid-based signal makes them a good species in which to examine whether and how perceptual systems are matched to signal expression.

Categorical perception makes clear predictions regarding labeling and discrimination of stimuli (StuddertKennedy et al. 1970). To demonstrate labeling, one tests individuals' abilities to discriminate between variants when comparing one end point of a stimulus continuum to variants occurring at increasing distances along that continuum. Categorical perception predicts that at a certain point along the continuum - the putative category boundaryindividuals change from labeling two stimuli as "similar" to labeling them as "different," which in nonhuman animal studies is represented by an abrupt increase in the ability to tell two stimuli apart. Importantly, this putative boundary should be found at the same point on the continuum regardless of which end point the test stimulus is being compared to. Labeling tests thus allow one to generate a hypothesis regarding the location of a category boundary. The second requirement is a significant increase in the ability to discriminate between two stimuli from opposite sides of the hypothesized boundary relative to two equally different stimuli drawn from the same side of the boundary.

We used behavioral assays to test for labeling and discrimination in Bengalese finches along the same orangered carotenoid color continuum previously tested in zebra finches (Caves et al. 2018). Counter to what has been found in zebra finches, our labeling trials did not show evidence of a category boundary, and discrimination trials pointed to differences in brightness between two colors as a better predictor of discrimination ability in Bengalese finches than a category boundary. Therefore, we further assayed color discrimination ability in both species using an additional set of colors that provided us with data on discrimination ability for color pairs that differed systematically in both hue (i.e., how red or orange they were) and brightness. These additional color discrimination assays provided data for predictive models that allowed us to examine the influence of hue, brightness, and (if present) categorical perception on color discrimination in each species.

\section{Methods}

Experimental Subjects

Subjects were sexually mature female Bengalese and zebra finches. Zebra finches were obtained from a colony maintained by Richard Mooney at Duke University (Institutional Animal Care and Use Committee [IACUC] protocol A258-14-10). Bengalese finches were obtained from Magnolia Bird Farm (Riverside, CA). Female Bengalese finches were identified using polymerase chain reaction (PCR) following the methods described in Griffiths et al. (1998).

Birds were housed in individual cages $(12 \mathrm{~cm} \times 18 \mathrm{~cm} \times$ $13 \mathrm{~cm}$; Prevue Pet) outfitted with perches, a cuttlebone, and ad lib. water and seed (Kaytee Forti-Diet Pro Health Finch diet). Outside of trials, lighting was provided by fluorescent bulbs (Ecolux with Starcoat SP 35/41, color temperature 3,500-4,100 K; General Electric) with ballast (Hi-Lume 3D/Eco-10; Lutron Electronics) operating at $60 \mathrm{~Hz}$ and kept on a 15L:9D photoperiod. Rooms were maintained at $25^{\circ}-27^{\circ} \mathrm{C}$. All methods were approved under Duke University IACUC protocol A004-17-01.

\section{Color Stimuli}

To assess color discrimination ability, we followed methods described in Caves et al. (2018). In brief, previous work showed that the beak colors of male zebra finches can be approximately represented by red and orange colors in the Munsell color system. Thus, we used as a starting point a set of 40 Munsell colors previously identified as capturing the variation in male zebra finch beak coloration (Burley and Coopersmith 1987; Collins et al. 1994; Birkhead et al. 1998). We measured reflectance spectra from each of these 40 colors (Munsell color paper; Pantone, Carlstadt, NJ) using an integrating sphere with a built-in tungstenhalogen light source (ISP-REF integrating sphere; Ocean Optics, Dunedin, FL), relative to a Labsphere Spectralon 99\% white standard.

For each measured color, we calculated photon catches, which quantify the relative stimulation of each photoreceptor type in response to viewing a certain stimulus. In contrast to primates, birds are thought to have separate sets of photoreceptors for encoding brightness information (double cones) and color information (single cones; Osorio 
and Vorobyev 2005). Thus, we quantified relative photon catches for both the double and the single (ultraviolet-, short-, medium-, and long-wavelength) cones as measures of perceived brightness and color, respectively. Relative photon catches were calculated over wavelengths from 400 to $700 \mathrm{~nm}$ (table S1; tables S1-S5 are available online), using the following formula:

$$
Q_{r, c}(\lambda) \propto \int_{400}^{700} S_{r}(\lambda) \times R_{c}(\lambda) \times I(\lambda) d \lambda,
$$

where $Q$ is the photon catch for photoreceptor type $r$ in response to color $c, S_{r}$ is the spectral sensitivity (i.e., the sensitivity to light of different wavelengths) in zebra finches of photoreceptor type $r$ (data from Lind 2016), $R_{c}$ is the reflectance of color $c, \lambda$ denotes wavelength, and $I$ is the irradiance of the ambient illuminant. As an ambient light spectrum, we used the CIE Illuminant A standard tungsten bulb illuminance spectrum (color temperature: $2,856 \mathrm{~K}$ ). Illuminant A is nearly identical to the actual ambient light in our experimental room (fig. S1; figs. S1-S3 are available online), and predicted color discriminability under standard and experimental lighting conditions were nearly identical (table S2).

We used the log of the photon catch values to calculate measures of the brightness and hue differences between color pairs. As a measure of the brightness difference between two colors, we calculated the Michelson contrast (Cronin et al. 2014), which is the ratio of the difference to the sum of the double-cone quantum catches $\left(Q_{1}-\right.$ $\left.Q_{2} / Q_{1}+Q_{2}\right)$. For hue difference, we calculated chromatic distance $(\Delta S$, a measure of the predicted discriminability between two colors); $\Delta S$ was calculated using the receptor noise-limited (RNL) model of color discrimination (Vorobyev and Osorio 1998), which assumes that the ability to discriminate two colors is limited by noise in the photoreceptors. We then visualized $\Delta S$ in a perceptually uniform, two-dimensional space based on hue and saturation (also known as chroma, or color intensity) in which the Euclidean distance between two colors is equivalent to the RNL model-derived chromatic distance (equations for chromaticity space are in Hempel de Ibarra et al. 2001).

This chromaticity space can be used for trichromatic vision (visual systems in which three cone types convey color information). Although birds have four cone types that contribute to color vision, the use of trichromatic vision under our particular experimental conditions is appropriate because, under our experimental lighting, the reflected ultraviolet (UV) radiance - and thus the contribution to color vision from the UV cone-is essentially zero (fig. S1). Thus, the quantum catch for the UV cone was on average $( \pm S D)$ only $0.26 \% \pm 0.10 \%$ (range: $0.14 \%$ to $0.42 \%$ ) of total single cone quantum catch (see table S1). Recalculating $\Delta S$ using a tetrachromatic visual system (i.e., including the UV cone catch) had minimal impact on predicted discriminability (table S2), changing $\Delta S$ values by a mean $( \pm S D)$ of $0.26 \pm 0.41$ (range: -0.18 to 0.99 ). Therefore, we expect that the impact of the UV cone on color perception was minimal and did not include the UV photon catch in our calculations.

Assuming a trichromatic visual system also allowed us to visualize the relative positions of all 40 Munsell color swatches that we measured by their $X$ and $Y$ coordinates in the chromaticity space described above, in which Euclidean distance is equivalent to $\Delta S$ (Hempel de Ibarra et al. 2001). We used those coordinates to select two sets of colors for use in behavioral experiments (fig. 1). The first set (the "beak set") was a set of eight colors, previously used in Caves et al. (2018), chosen for two reasons. First, those colors span the full range of colors previously used to describe male zebra finch beaks (Burley and Coopersmith 1987; Collins et al. 1994; Birkhead et al. 1998). Second, those colors are approximately equally spaced in the chromaticity space described above that is based on zebra finch spectral sensitivity, and thus they are predicted to be equally discriminable from one another, based on chromatic cues. As with real beak colors, the colors in the beak set also varied in perceived brightness. Female zebra finches

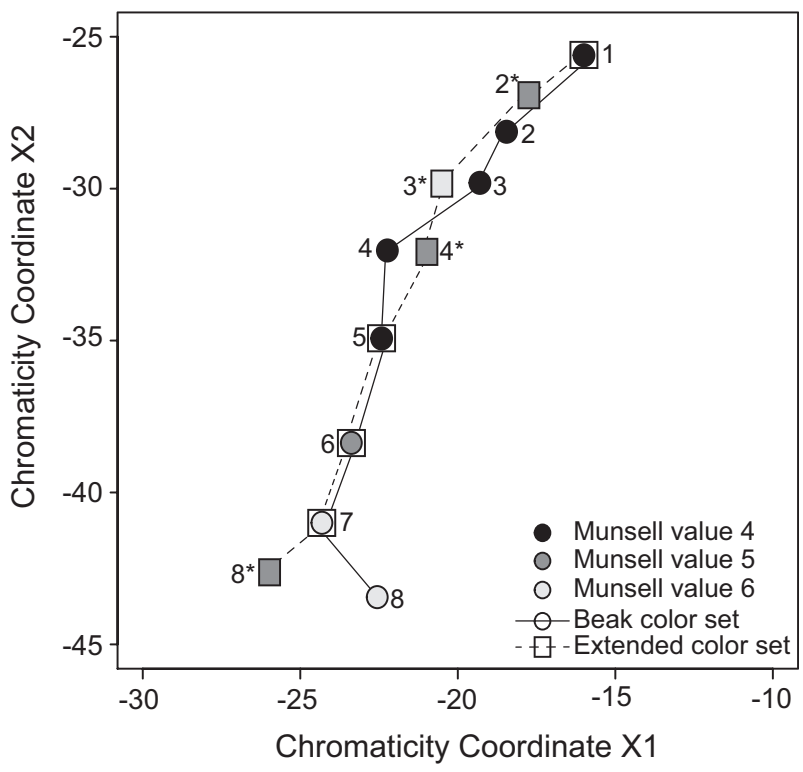

Figure 1: Locations of selected colors in avian chromaticity space. Circles and solid lines indicate the beak set, while squares and dashed lines indicate the extended set. The gray shading of an icon indicates its Munsell value. Colors range from 1 (the most red) to 8 (the most orange), and numbers marked with an asterisk are the four new colors that were used in the extended set. 
categorically perceive the beak set, with a boundary between colors 5 and 6 (Caves et al. 2018).

The second set (the "extended set") was chosen to provide further information about the relative effects of chromatic distance and Michelson contrast on discrimination ability. Behavioral color discrimination data from the extended set were used as training data for a predictive model of color discrimination ability (for details, see "Statistical Analyses"). To create the extended set, we replaced four colors in the beak set with new colors, with the resultant extended set spanning the same range in color space and in brightness and having roughly the same chromatic distance between neighboring colors as the beak set (fig. 1). However, rather than steadily increasing in brightness, the extended colors each differed from their neighboring colors by a Munsell value (the measure of brightness in the Munsell system) of 1 . Colors 5 and 6-which span the previously described location of the category boundary in the beak set-were the same in the two color sets.

Throughout, relative photon catches - and thus brightness and $\Delta S$ - were calculated using spectral sensitivity curves for the zebra finch (Bowmaker et al. 1997; Lind 2016) because spectral sensitivity curves for Bengalese finches are not available. However, both species have UVS visual systems (one of two types of color vision systems found in birds; Hart 2001), and variance in peak spectral sensitivities of both single and double cones across estrildid finches is extremely low (fig. S2; Hart et al. 2000), so it is likely that Bengalese and zebra finches have similar spectral sensitivities. Additionally, recalculating $\Delta S$ using a different UVS visual system (the starling, Sturnus vulgaris), the average UVS cone-type retina, or the average VS cone-type retina, which is the other primary type of retina found in birds (data from Endler and Mielke 2005), had little impact on relative chromatic distances (table S2). Thus, $\Delta S$ is robust even to large differences in spectral sensitivity for this set of colors and so is unlikely to differ substantially between Bengalese and zebra finches.

\section{Behavioral Tests of Color Discrimination}

For both the beak set and the extended set, we created disk stimuli by gluing together two semicircular halves of Munsell paper to form a circle. The two halves were either the same color ("solid") or different colors ("bicolor") and covered with a clear epoxy cover. We tested color discrimination using a food-reward protocol in which birds were presented with a foraging grid containing 12 wells, six of which were covered by the disk stimuli described above, two by bicolor disks, and four by solid disks (two of each color comprising the bicolor disks). We trained birds to search for food rewards beneath bicolor disks made of the two end point colors from the beak set, 1 and 8 (i.e., $1 \mid 8$ ).
Birds passed a trial if they removed both bicolor disks before any solid disks, which would occur by chance in only 1 of 15 trials. We gave birds 2 min to pass each trial. Birds that passed six of seven consecutive training trials began experimental trials.

In experimental trials, the makeup of the disks on the grid was the same as in the training trials, but we varied the two colors comprising the disks. Two types of trials were run: labeling and discrimination. Labeling trials used bicolor disks that included the end point colors in combination with all other colors (e.g., $1|2,1| 3,1 \mid 4$, etc. and $8|7,8| 6,8 \mid 5$, etc.). In discrimination trials, disks comprised color combinations that were equally spaced across the continuum, meaning that they were one (i.e., $1|2,2| 3,3 \mid 4$, etc.), two (i.e., $1|3,2| 4,3 \mid 5$, etc.), or three (i.e., $1 \mid 4$, $2 \mid 5$, $3 \mid 6$, etc.) color steps apart, referred to below as "one-apart," "two-apart," and "three-apart," respectively.

On experimental trial days, we removed food at 0900 hours, to ensure that birds were motivated to perform the task, and began trials at 1400 hours. During trials, lighting was provided by halogen bulbs (color temperature: 2,900 K; model number: H\&PC-61361; Philips Lighting) hung approximately $80 \mathrm{~cm}$ above the cage and filtered through vellum paper to provide diffuse lighting. Birds were allowed at least 5 min to acclimate to the experimental lighting conditions before trials began.

To assess whether female Bengalese finches perceive carotenoid-based coloration categorically, as zebra finches do (Caves et al. 2018), we first ran labeling and discrimination trials on female Bengalese finches $(n=10)$ using the beak set. To further assess the relative contributions of chromatic distance and Michelson contrast to discrimination ability, we performed discrimination trials using the same Bengalese finches $(n=10)$ and the extended set of colors. Finally, to compare zebra finch perception with Bengalese finch perception across both color ranges, we performed discrimination trials on female zebra finches $(n=8)$ using the extended set. Data on female zebra finch labeling and discrimination of the beak set $(n=26)$ were taken from Caves et al. (2018).

As in Caves et al. (2018), we also performed trials in which the disks were the same color on each half $(1 \mid 1$ or $8 \mid 8$ ) to control for the possibility that birds detected seeds by olfaction. In both cases birds performed no better than chance, indicating that they did not use olfactory cues in determining which disks to flip. These olfactioncontrol comparisons are not included in further analysis. We randomized the location of disks on the grid for each trial using the sample function in $\mathrm{R}$ ( $\mathrm{R}$ Development Core Team 2019). For each bird, we performed 10 trials for each color combination (all individuals participated in at least five trials for each color combination) and calculated "pass frequency," the proportion of trials they 
passed for a given color combination. Throughout, we use pass frequency as a measure of color discrimination ability. Each day, trials began with a refresher, in which birds were given one trial with $1 \mid 8$ disks to ensure they remembered the trained task, and ended with a motivation check, in which we recorded the amount of time it took birds to begin eating seeds out of their regular dish once it was returned to the cage to ensure that birds had remained hungry and motivated throughout the task.

\section{Statistical Analyses}

Data used for all analyses are available in the Dryad Digital Repository (https://doi.org/10.5061/dryad.stqjq2c1z; Caves et al. 2020). We found no evidence for categorical perception in Bengalese finches (see "Results"), but Michelson contrast appeared to play an important role in discrimination ability. This finding was different from prior results for zebra finches, in which a color category boundary best explained color discrimination ability. To statistically explore the relative impacts of a color category boundary or Michelson contrast on color discrimination ability, we built linear mixed models (using lme4; Bates et al. 2015) to describe the average pass frequency for each type of discrimination task (one-apart, two-apart, or three-apart) for each species. We used lmerTest (Kuznetsova et al. 2017) to estimate degrees of freedom and calculate $P$ values via Satterthwaite's method. As the response variable, we used the pass frequency for each bird for each color combination. Predictor variables were either a binary indicator of whether a given comparison crossed the 5-6 boundary (the "category" model) or Michelson contrast between color pairs (the "contrast" model). For the models that considered one-apart, two-apart, or three-apart data on their own, we did not include chromatic distance as a predictor because all comparisons within a discrimination set are approximately equally spaced by design. However, we also built category and contrast models that included all discrimination data (one-apart, two-apart, and three-apart) and the predictor of chromatic distance.

Model fit was assessed using the Akaike information criterion (AIC), a value that can be used to determine which of a set of models is most likely to be the best model for a given data set (Burnham and Anderson 2002 and references therein). Change in AIC value $(\triangle \mathrm{AIC})$ was calculated relative to the better-fitting model in each case, and following Burnham et al. (2011) $\Delta$ AIC values were used to calculate relative likelihoods for each model $i$ within a set using the formula

$$
l_{i}=\exp \left[-(1 / 2) \Delta \mathrm{AIC}_{i}\right]
$$

We then calculated the Akaike weight for each model by dividing the likelihood of a given model $i$ by the sum of the likelihoods of all models within that set (Burnham et al. 2011). The Akaike weight gives the probability that each model within a set of models is the best model.

To further examine differences between Bengalese finches and zebra finches in their color discrimination abilities, we built a model to predict how discriminable a color pair should be for each species depending on chromatic distance and Michelson contrast. We built this model using pass frequency data from the extended set because color comparisons in that set had been chosen to vary systematically in both chromatic distance and Michelson contrast (the extended set colors were the same for both species, but separate predictive models were built for Bengalese and zebra finches). We then tested how well data from the beak set matched the predictive model built from the extended set. We specifically wanted to examine how pass frequencies for comparisons that do or do not cross the 5-6 boundary in the beak set were predicted by chromatic distance and Michelson contrast (i.e., brightness differences) in both Bengalese finches and zebra finches. Thus, to avoid circularity that would result from building the model using data on cross-boundary comparisons that it would then be used to predict, we built the model using the 13 comparisons from the extended set that did not include the original 5-6 boundary (i.e., no comparisons used in the model included the color step between colors 5 and 6; table S3).

These predictive models (one for each species) were fixed-intercept linear models that included pass frequency as the response variable and both chromatic distance and Michelson contrast as predictors. The intercept was set to the probability of a bird passing a task by chance (i.e., 1/15). We found that the predictive models well described the data used to construct them. In each species, the predictive model that included both terms performed better than did a model that included only chromatic distance or Michelson contrast (table S5). Specifically, in both species pass frequency for the extended set was strongly predicted by the combination of chromatic distance and Michelson contrast (for Bengalese finches, $R^{2}=0.96$; for zebra finches, $R^{2}=0.91$ ), and coefficient estimates were similar in both species (see table S4).

The coefficients from the predictive model represented expected pass frequency for color combinations of a given chromatic distance and Michelson contrast. We then visualized how observed pass frequency on discrimination tasks from the beak set aligned with the expected pass frequency values generated by the model by plotting observed versus expected pass frequency. In this plot, points falling on the line of slope 1 were those in which observed pass frequency was perfectly aligned with expected pass frequency (i.e., perfectly predicted by chromatic distance and Michelson contrast). 
Last, we built linear models for each species of observed pass frequencies for color comparisons in the beak set as predicted by (1) expected pass frequency, (2) whether the beak set comparison included the 5-6 boundary, and (3) the interaction between these terms. When categorical perception is operating, the combination of chromatic distance and Michelson contrast should have different effects on discrimination of comparisons that cross the category boundary compared with those that do not. We therefore predicted that the interaction term in the model (between expected pass frequency and crossing the 5-6 boundary) should be significant only if a species exhibits categorical perception.

\section{Evaluating Sample Size Differences between Bengalese Finches and Zebra Finches}

To ensure that the apparent lack of categorical perception in Bengalese finches was not simply the result of having a smaller sample size of Bengalese finches than in the original zebra finch study (Caves et al. 2018), we performed a resampling analysis in which we used linear mixed effects models to calculate the effect of each color step on pass frequency (for details, see fig. S3). We randomly resampled 10 individuals (the sample size of Bengalese finches) from the original zebra finch data set $(n=26) 1,000$ times and for each permutation calculated the effect of each color step on pass frequency. This analysis showed clearly that even if the sample size of zebra finches was only 10 , the effect of the $5-6$ color step is larger than that of any other color step, as is consistent with categorical perception at the 5-6 boundary, in all but six of 1,000 permutations (fig. S3). Thus, a sample size larger than 10 individuals is not required to detect the signature of categorical perception.

\section{Results}

\section{Bengalese Finches Show No Evidence of Categorical Perception of Carotenoid Coloration}

We first asked whether Bengalese finches label the beak set of colors in a way that is consistent with a boundary between colors 5 and 6 , as was found in zebra finches. In Bengalese finches, pass frequency increased with increasing chromatic distance from the end point colors 1 or 8 . Thus, color discrimination improves as color pairs become more distinct from one another, with the mean $( \pm S D)$ increase in pass frequency between neighboring colors (e.g., the increase in pass frequency from $1 \mid 2$ to $1 \mid 3$ or from $1 \mid 3$ to $1 \mid 4$ ) being $0.12 \pm 0.09$. Across the color range, pass frequency increased more between some color pairs than others, but in order for labeling to indicate a potential category boundary, the largest increase should occur between the same colors in each direction. However, we observed that for comparisons that included color 1, the largest increase occurred between $1 \mid 3$ and $1 \mid 4(0.25)$, while for comparisons that included color 8 , the largest increase in pass frequency occurred between $6 \mid 8$ and $5 \mid 8$ (0.25; fig. $2 A$ ). Thus, the labeling data did not suggest the presence of a category boundary along the continuum. Additionally, the increase in pass frequency from $1 \mid 5$ to $1 \mid 6(0.12)$ was smaller than that from $1 \mid 7$ and $1 \mid 8(0.22)$ and on par with the increase between $1 \mid 6$ and $1 \mid 7(0.10)$, further indicating that the 5-6 color step, the location of a category boundary in zebra finches (fig. $2 B$ ), does not have special significance for the Bengalese finches.

The lack of evidence for a category boundary in the labeling data suggests that Bengalese finches do not exhibit categorical perception within the beak set color range. Despite this, during discrimination trials (those that use equally spaced color pairs from across the continuum rather than comparisons with end point colors), Bengalese finches exhibited higher pass frequencies for some equally spaced color combinations than others. In particular, Bengalese finch ability to discriminate between two colors generally increased as the Michelson contrast between two colors increased (fig. 3). In support of this, in Bengalese finches the contrast model, in which the predictor variable was the Michelson contrast of a given color comparison, was a substantially better fit than the category model. This result held whether considering color combinations that were one, two, or three color steps apart as well as for all comparisons combined (table 1). In zebra finches, however, the category model, in which the predictor variable was a binary indicator of whether a comparison crossed the 5-6 boundary, was a better fit in all cases, in line with our previous findings of categorical perception in this species.

In summary, for Bengalese finches (1) labeling did not indicate the presence of a category boundary, (2) the pattern of increased discrimination for some color pairs over others appears largely attributable to differences in contrast, and (3) models suggested that contrast described the data better than did a category boundary, unlike in zebra finches.

\section{Contribution of Chromatic Distance and Michelson Contrast to Color Discrimination}

To further examine differences in how Bengalese and zebra finches discriminate colors, we used pass frequency data from the extended set of colors to build models in which pass frequency was predicted by chromatic distance and Michelson contrast in each species. We then applied the model fit to observed pass frequency in the beak set in two ways. First, we visually examined their relationship to observed pass frequencies (fig. 4). Second, 


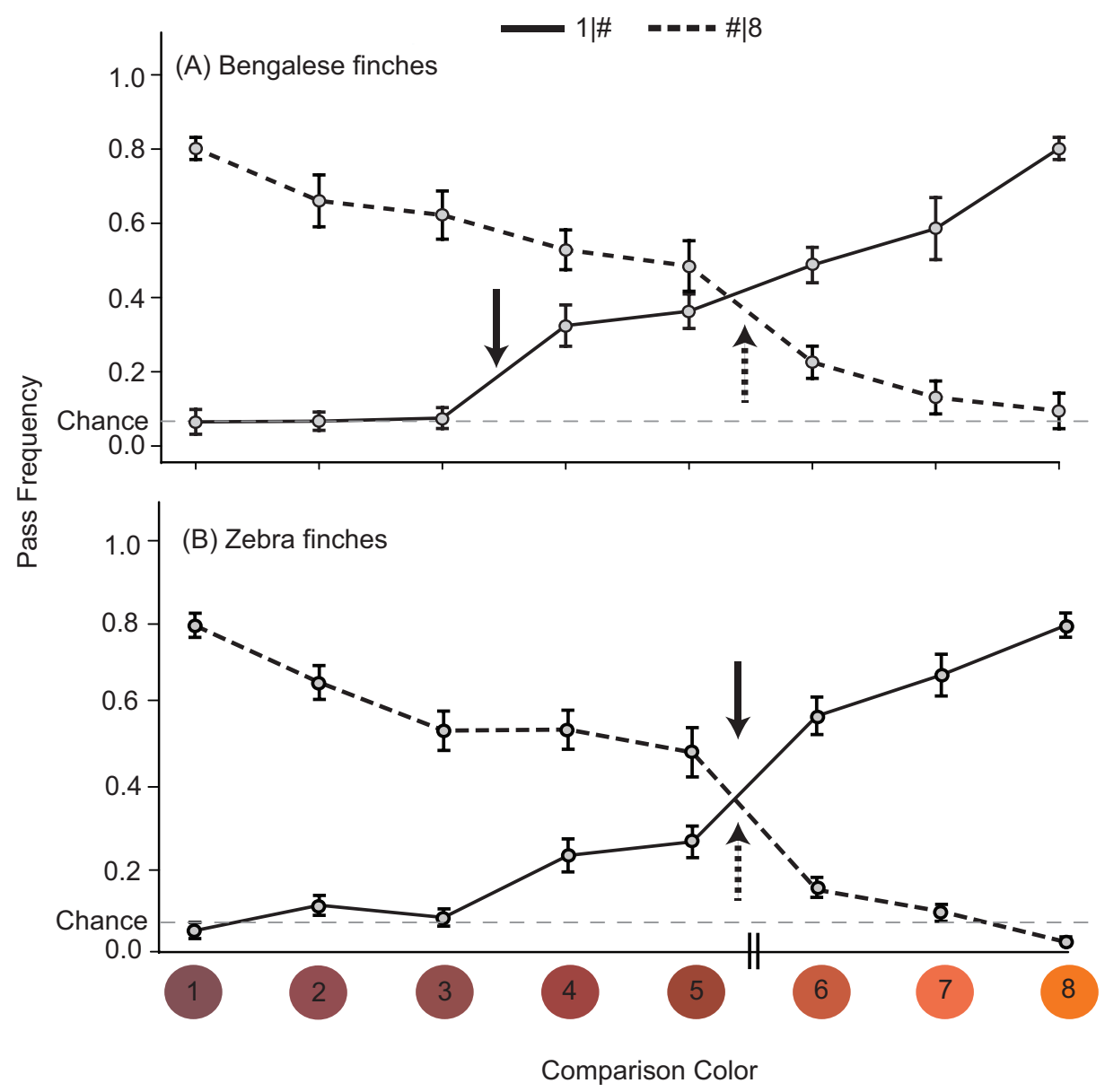

Figure 2: Labeling data for the beak set in Bengalese finches $(A)$ and zebra finches $(B)$. A, Female Bengalese finches showed no evidence of labeling consistent with categorical perception in that the largest increases in pass frequency (arrows) occurred in different parts of the color continuum in each direction. $B$, Female zebra finches showed the characteristic labeling curve for categorical perception, with the largest increases in pass frequency (arrows) being in the same place, between colors 5 and 6, in each direction (data from Caves et al. 2018). The solid line represents pass frequency for $1 \mid$ \# trials, and the dashed line represent results for \# $\mid 8$ trials. Vertical bars with each circle represent standard errors. The dashed gray line indicates expected pass frequency if birds flip disks at random.

we used a modeling approach to examine how observed color discrimination ability in each species is predicted by pass frequency for a given comparison and whether that comparison included the 5-6 boundary, as well as the interaction between those terms (table 2).

In Bengalese finches, observed pass frequency for all of the beak set color discrimination tasks was strongly correlated with expected pass frequency from the predictive model (i.e., the combination of chromatic distance and Michelson contrast), regardless of whether comparisons crossed the 5-6 boundary reported in zebra finches (fig. $4 A$, black line and points) or not (fig. $4 A$, gray solid line and points). There were nearly identical slopes of the lines for comparisons that either crossed (slope: 0.86) or did not cross (slope: 0.85 ) the 5-6 boundary, showing that the re- lationship between observed pass frequency and the pass frequency predicted by chromatic distance and contrast was the same for within- and between-category comparisons. Furthermore, a model showed that expected pass frequency explained a similar amount of variance regardless of whether the comparison crossed the boundary (for crosses, $R^{2}=0.79$; for does not cross, $R^{2}=0.64$ ). Therefore, though expected pass frequency strongly predicted observed pass frequency, the interaction between expected pass frequency and whether a comparison crosses the 5-6 boundary was not significant (table 2).

By comparison, in zebra finches we found clear differences between within- versus cross-boundary comparisons in terms of whether pass frequency was predicted by chromatic distance and Michelson contrast (fig. $4 B$ ). The 


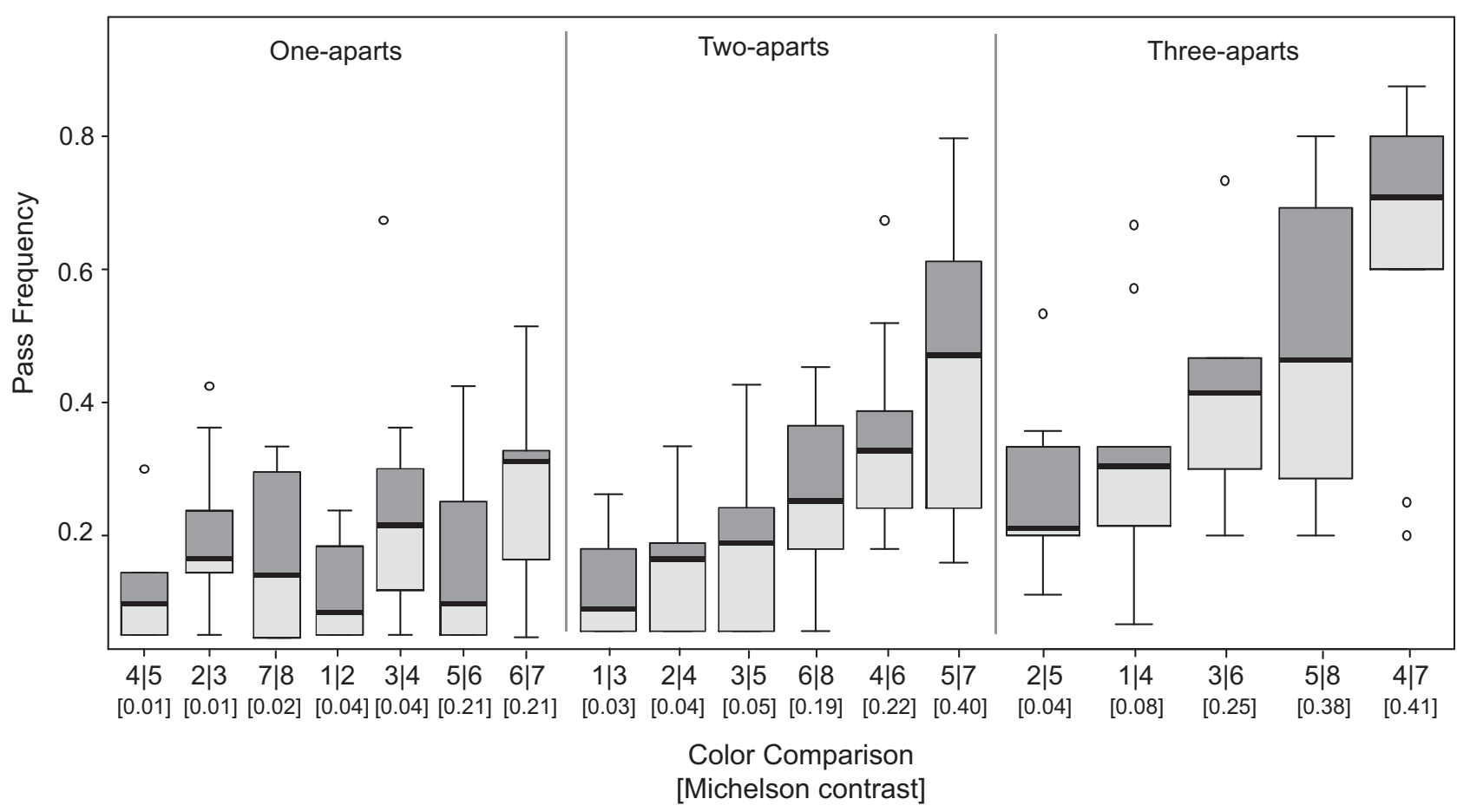

Figure 3: Bengalese finch discrimination of beak set color stimuli that are one, two, or three color steps apart. Within each comparison type, boxes are ordered from left to right by increasing Michelson contrast (shown in brackets). For each comparison, the median (horizontal line), 25th and 75th percentiles (boxes), and $1.5 \times$ interquartile range (whiskers) for individual pass frequency are presented.

slope of the line for comparisons that crossed (slope: 0.99) was different from the slope of the line for comparisons that did not cross the boundary (slope: 0.64 ). Models showed pass frequency on cross-boundary comparisons (fig. $4 B$, black line and points) was predicted very well by chromatic distance and Michelson contrast $\left(R^{2}=0.92\right)$, whereas pass frequency for within-category comparisons (fig. $4 B$, gray line and points) was not explained by the same relationship between chromatic distance and Michelson contrast $\left(R^{2}=0.19\right)$. Additionally, the interaction term between expected pass frequency and crossing the 5-6 boundary was significant $(P=.04$; table 2$)$. This indicates a significant effect of crossing the boundary on how well color discrimination ability can be predicted by Michelson contrast and chromatic distance.

Overall, we found clear differences between Bengalese finches and zebra finches in whether their ability to discriminate between color pairs that do or do not cross the 5-6 boundary was predicted by chromatic distance and Michelson contrast. In particular, Bengalese finch color discrimination was predicted well by chromatic distance and Michelson contrast for both cross-boundary and withincategory comparisons. In zebra finches, however, whether the combination of chromatic distance and Michelson contrast predicted pass frequency depended on whether that comparison involved colors from opposite sides of the 5-6 boundary.

\section{Discussion}

Our results show differences in orange-red color perception in two related estrildid finches that likely have very similar spectral sensitivity (given the highly conserved spectral sensitivities of the photoreceptors within the family Estrildidae; fig. S2; Hart et al. 2000) but that differ in their use of color in signaling. In female Bengalese finches, tests of color discrimination ability showed no evidence of categorical perception of orange-red coloration. Labeling tests did not indicate a category boundary (fig. 2), and variation in discrimination of equally spaced color pairs was explained better by the Michelson contrast between colors than the presence of a category boundary (fig. 3; table 1). This finding stands in contrast to female zebra finches, in which categorical perception of this same color range has been previously demonstrated (Caves et al. 2018) and in which discrimination of equally spaced color pairs was better explained by a category boundary than by differences in Michelson contrast (table 1). This finding is consistent with the hypothesis that perceptual processes may be adapted for signal function. In this case, zebra finches categorically 
Table 1: Model fits for linear mixed models that predict pass frequency on one-, two-, and three-apart discrimination tasks using either the Michelson contrast or a binary indicator of whether that comparison crosses the 5-6 boundary found in zebra finches and include bird ID as a random effect

\begin{tabular}{|c|c|c|c|c|c|c|}
\hline & $R^{2}$ & AIC & $\Delta \mathrm{AIC}$ & $l_{i}$ & $w_{i}$ & Interpretation \\
\hline \multicolumn{7}{|l|}{ Zebra finches: } \\
\hline \multicolumn{7}{|l|}{ One-apart: } \\
\hline Category & .4 & -15.7 & 0 & 1.0 & .60 & \multirow[t]{2}{*}{ Categorical model better } \\
\hline Contrast & .33 & -14.9 & .8 & .67 & .40 & \\
\hline \multicolumn{7}{|l|}{ Two-apart: } \\
\hline Category & .90 & -15.3 & 0 & 1.0 & .96 & \multirow[t]{2}{*}{ Categorical model better } \\
\hline Contrast & .70 & -8.7 & 6.6 & .04 & .04 & \\
\hline \multicolumn{7}{|l|}{ Three-apart: } \\
\hline Category & .99 & -24.6 & 0 & 1.0 & .99 & \multirow[t]{2}{*}{ Categorical model better } \\
\hline Contrast & .92 & -13.3 & 11.3 & $<.01$ & $<.01$ & \\
\hline \multicolumn{7}{|c|}{ Combined model: } \\
\hline Category & .84 & -46.2 & 0 & 1 & .91 & \multirow[t]{2}{*}{ Categorical model better } \\
\hline Contrast & .80 & -41.6 & 4.6 & .1 & .09 & \\
\hline \multicolumn{7}{|c|}{ Bengalese finches: } \\
\hline \multicolumn{7}{|c|}{ One-apart: } \\
\hline Contrast & .41 & -17.5 & 0 & 1.0 & .86 & \multirow[t]{2}{*}{ Contrast model better } \\
\hline Category & .005 & -13.9 & 3.6 & .17 & .14 & \\
\hline \multicolumn{7}{|l|}{ Two-apart: } \\
\hline Contrast & .95 & -20.8 & 0 & 1.0 & .99 & \multirow[t]{2}{*}{ Contrast model better } \\
\hline Category & .79 & -11.6 & 9.2 & .01 & .01 & \\
\hline \multicolumn{7}{|l|}{ Three-apart: } \\
\hline Contrast & .88 & -10.5 & 0 & 1.0 & .85 & \multirow[t]{2}{*}{ Contrast model better } \\
\hline Category & .76 & -7.0 & 3.5 & .17 & .15 & \\
\hline \multicolumn{7}{|c|}{ Combined model: } \\
\hline Contrast & .87 & -43.2 & 0 & 1.0 & .99 & \multirow[t]{2}{*}{ Contrast model better } \\
\hline Category & .74 & -31.2 & 12.0 & $<.01$ & $<.01$ & \\
\hline
\end{tabular}

Note: The combined models contain data from all discrimination trials (one-, two-, and three-aparts) and additionally include chromatic distance as a fixed effect predictor. For each model pair, the better-fit model is listed first. $\Delta$ AIC is calculated relative to the best-fit model, $l_{i}$ is the relative likelihood of model $i$, and $w_{i}$ is the probability that it is the best model within a set. AIC = Akaike information criterion.

perceive an orange-red range of colors that serve an important signal function in mate choice (Burley and Coopersmith 1987; de Kogel and Prijs 1996; Blount et al. 2003), whereas Bengalese finches, which do not exhibit carotenoid-based coloration and thus are unlikely to use orange-red coloration in a signaling context, do not exhibit categorical perception of this color range. Given that this is only a twospecies comparison, a broader phylogenetic comparison (see, e.g., fig. S2) will be necessary to better understand the evolutionary implications of these results, specifically the extent to which the expression of categorical perception in estrildid finches is linked to the use of color signals. A concordance between the use of color signals and the expression of categorical perception would support the hypothesis that the expression of categorical perception evolves as an adaptive response to the costs of assessing continuously varying traits (Green et al. 2020).

Comparing color discrimination between zebra finches and Bengalese finches yields some insight into the potential mechanisms underlying categorical color perception.
In Bengalese finches, pass frequency on all color discrimination tasks was predicted by both chromatic distance and Michelson contrast (fig. 4A). In zebra finches, how well pass frequency was predicted by chromatic distance and Michelson contrast differed between within-category and cross-boundary comparisons (fig. 4B). Thus, in zebra finches it appears that the same sensory inputs - that is, the same differences in hue and brightness-lead to different behavioral outputs depending on where two colors lie relative to the category boundary. For zebra finches, in nine of 13 within-category comparisons observed pass frequency was lower than predicted (fig. $4 B$ ), suggesting that categorical perception suppresses within-category discrimination and enhances cross-boundary discrimination. By comparison, observed pass frequencies were higher than predicted for 11 of 12 between-category comparisons. This finding is interesting in light of a recent study examining how the carotenoid concentration of oil droplets found in zebra finch photoreceptors relates to categorical perception. In particular, individuals with a higher concentration 


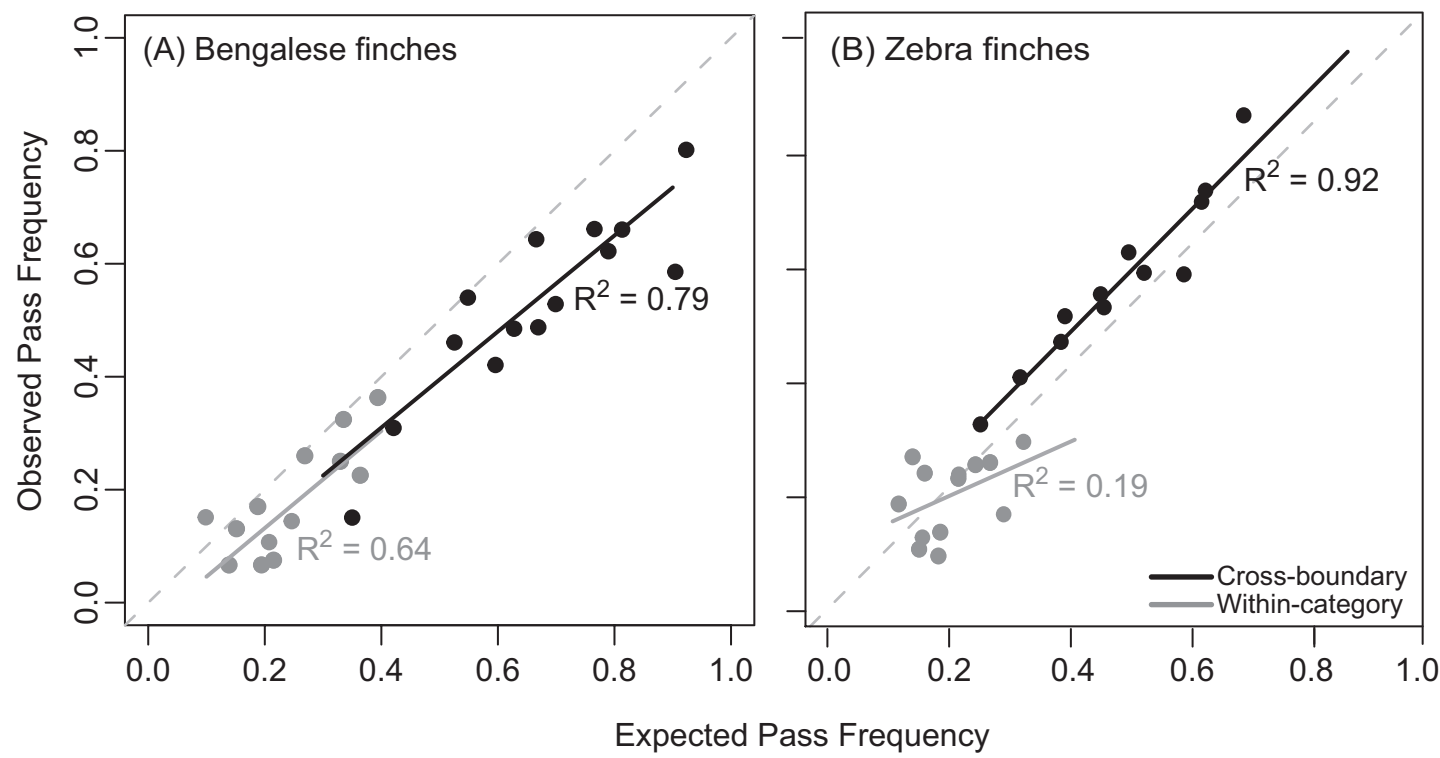

Figure 4: Observed pass frequency versus expected pass frequency for comparisons that do (black) and do not (gray) cross the 5-6 boundary in Bengalese $(A)$ and zebra $(B)$ finches. Each point is a color comparison from the original beak set. The dashed line has a slope of 1 (i.e., it represents the expected relationship if observed and expected pass frequencies are identical).

of carotenoids in their retinal oil droplets exhibited higher pass frequency for cross-boundary color discriminations but not for within-category discriminations (Caves et al. 2020). Together, these results suggest that categorical perception may suppress color discrimination within categories and that aspects of retinal physiology, such as oil droplet carotenoids, may serve as a mechanism by which to enhance cross-boundary discrimination.

Filtering by carotenoid-containing oil droplets, however, has only a small impact on categorical perception:
Caves et al. (2020) found that less than $20 \%$ of variation in cross-boundary color discrimination ability between individuals was attributable to variation in oil droplet carotenoids. More broadly, data increasingly show that predictions of color discrimination based on the spectral sensitivity of the photoreceptors alone (of which filtering by oil droplet carotenoids is a part) do not always align with behavioral color discrimination data (e.g., Caves et al. 2018; Cheney et al. 2019; Zipple et al. 2019). This is not surprising, given that many processes that occur after a stimulus is

Table 2: Relationship between expected and observed pass frequencies for comparisons that do or do not include the 5-6 boundary in Bengalese and zebra finches

\begin{tabular}{|c|c|c|c|c|}
\hline Species, parameter & Coefficient & SE & $P$ & Interpretation \\
\hline \multicolumn{5}{|l|}{ Bengalese finches: } \\
\hline Intercept & -.026 & & & \multirow{5}{*}{$\begin{array}{l}\text { Observed pass frequency is predicted by expected pass } \\
\text { frequency (i.e., chromatic distance plus Michelson } \\
\text { contrast) for all comparisons }\end{array}$} \\
\hline Expected pass frequency & .85 & .22 & .0008 & \\
\hline $5-6$ boundary & -.02 & .10 & .84 & \\
\hline $\begin{array}{l}\text { Interaction between expected } \\
\text { pass frequency and }\end{array}$ & & & & \\
\hline $5-6$ boundary & .007 & .25 & .98 & \\
\hline \multicolumn{5}{|l|}{ Zebra finches: } \\
\hline Intercept & .09 & & & \multirow{5}{*}{$\begin{array}{l}\text { Observed pass frequency is predicted by expected pass } \\
\text { frequency (i.e., chromatic distance plus Michelson } \\
\text { contrast) only for those comparisons that cross the } \\
5-6 \text { boundary }\end{array}$} \\
\hline Expected pass frequency & .44 & .23 & .08 & \\
\hline $5-6$ boundary & -.04 & .07 & .62 & \\
\hline $\begin{array}{l}\text { Interaction between expected } \\
\text { pass frequency and }\end{array}$ & & & & \\
\hline $5-6$ boundary & .56 & .26 & .04 & \\
\hline
\end{tabular}

Note: The 5-6 boundary term is a binary indicator of whether a given comparison included the 5-6 boundary. 
transduced by photoreceptors affect perception. Additionally, behavioral response to color signals can be plastic depending on the developmental environment, as has been shown in juvenile zebra finches raised by parents with manipulated bill coloration (ten Cate et al. 2006), although it is unclear whether perceptual processing or sexual preferences were altered in this case. Given that color preferences can be plastic, however, one line of future inquiry could investigate whether Bengalese finches possess the capacity to develop categorical perception if they are exposed to the relevant carotenoid-based colors in their developmental environment.

Our results may also contribute to our understanding of the mechanisms of color discrimination in birds and in particular lend new insight into a recent analysis performed by Price et al. (2019). In particular, Price and colleagues focused on zebra finch color discrimination data reported in Caves et al. (2018) for comparisons from the beak set that are two color steps apart (color comparisons $1|3,2| 4,3|5,4| 6,5 \mid 7$, and $6 \mid 8$ ). They noted a close fit between observed zebra finch discrimination data and the predicted discriminability of color pairs if zebra finch color discrimination is based on luminance contrast (i.e., the photon catch of the double cones). However, the zebra finch discrimination data deviate from the predictions based on luminance contrast close to the category boundary (fig. 5, gray vs. dashed lines). First, in the behavioral data from Caves et al. (2018), pass frequency increases less from $4 \mid 6$ to $5 \mid 7$ than the luminance model of Price et al. (2019) predicts because the luminance model predicts that birds should pass at much higher frequency for $5 \mid 7$ (Michelson contrast: $~ 0.4$ ) than for $4 \mid 6$ (Michelson contrast: $\sim 0.2$ ). Second, behavioral pass frequency decreases more from $5 \mid 7$ to $6 \mid 8$ than predicted by the luminance model, with behavioral pass frequency for $6 \mid 8$ (a relatively high-contrast comparison) on par with pass frequency for $1|3,2| 4$, and $3 \mid 5$, where Michelson contrast is approximately zero. These deviations from the expectations from Price et al. (2019) are consistent with our model results (table 1), which indicate that zebra finch color discrimination is better predicted by a category boundary than by luminance differences. Behavioral data from the Bengalese finches, however, in which brightness differences play a critical role in discrimination, line up very closely with luminance contrastbased predictions of discriminability from the Price et al. (2019) model (fig. 5, black line vs. dashed line). Thus, one possibility is that luminance contrast underlies color discrimination in birds but that processes like categorical perception can further modify how color is perceived.

In conclusion, the Bengalese finch, a species lacking carotenoid-based coloration, does not discriminate colors along an orange-red continuum in a categorical fashion.

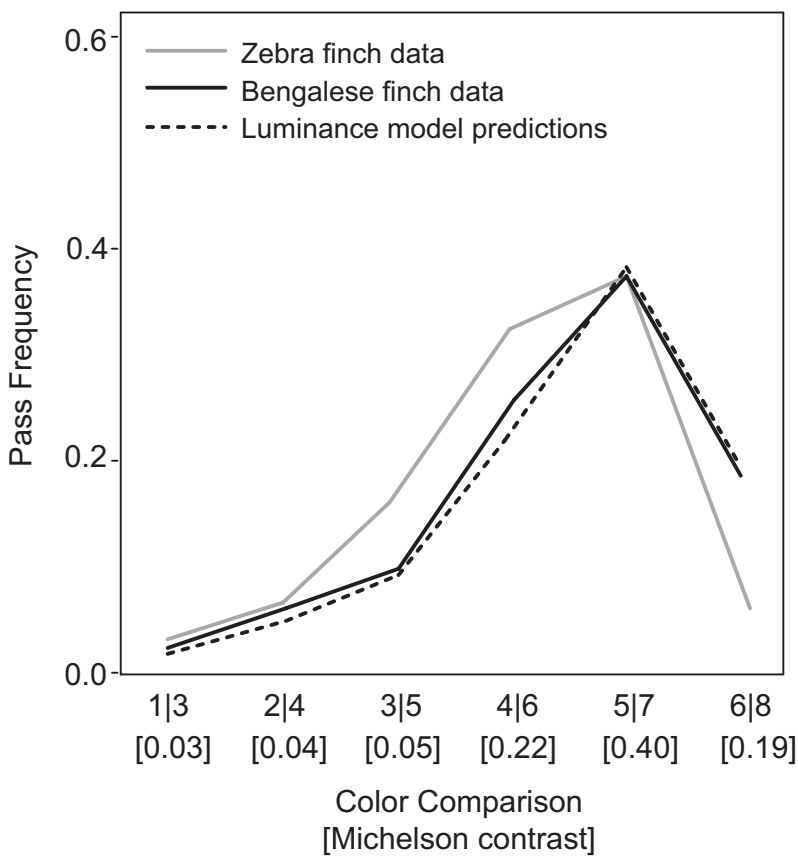

Figure 5: Behavioral color discrimination data for two-apart comparisons for zebra finches (solid gray line) and Bengalese finches (solid black line) as well as predicted pass frequency from a model of color discrimination based on luminance contrast information (dashed line). Data for zebra finches are originally from Caves et al. (2018), and data for Bengalese finches are presented here. The model predictions are redrawn from figure $4 b$ of Price et al. (2019), who state that the $Y$-axis values for their predictions are unknown because they depend on how the inputs from each photoreceptor type are weighted but that the shape of the predicted curve is of more importance than its height. Thus, their prediction curve is scaled here so that the maximum pass frequency aligns with the observed maximum mean pass frequency in our studies.

This is in contrast to the zebra finch, for which this color range plays an important role in signaling and which expresses categorical perception of colors along the same continuum. This finding is consistent with the idea that selection on a signaling system may act on the perceptual mechanisms underlying that system. However, a more comprehensive phylogenetic analysis of the relationship between signal expression and differences in perception is needed to better understand how perceptual processes evolve in the context of signaling and the ways in which signal receivers and senders may place selective pressures on one another.

\section{Acknowledgments}

We thank Trevor Price, Daniel Bolnick, J. Albert C. Uy, and two anonymous reviewers for helpful comments on the manuscript. We also thank Lindsey Kinsella and Megan 
Knauer for assistance with behavioral testing, Richard Mooney and Michael Booze for providing access to zebra finches from their colony, and Benjamin Wheeler, Ryan Campbell, Robert Fitak, Jelmer Poelstra, and Anne Yoder for access to facilities and help with PCR analyses. Funding for this study was provided by the Duke University Office of the Provost.

\section{Statement of Authorship}

E.M.C., P.A.G., S.P., S.J., and S.N. conceived the study. S.N. acquired funding. E.M.C., P.A.G., and S.J. developed the methods and designed the experiment. E.M.C., P.A.G., and D.B. collected the data. M.N.Z. led the data analysis, model analysis, coding, and visualization with E.M.C. S.P. and S.N. provided resources. E.M.C. wrote the original draft. All authors reviewed and edited the manuscript.

\section{Data and Code Availability}

Data and code supporting the results are archived in the Dryad Data Repository (https://doi.org/10.5061/dryad .stqjq2c1z; Caves et al. 2020).

\section{Literature Cited}

Bates, D., M. Maechler, B. Bolker, and S. Walker. 2015. Fitting linear mixed-effects models using lme4. Journal of Statistical Software 67:1-48.

Birkhead, T. R., F. Fletcher, and E. J. Pellatt. 1998. Sexual selection in the zebra finch Taeniopygia guttata: condition, sex traits and immune capacity. Behavioral Ecology and Sociobiology 44:179-191.

Blount, J. D., N. B. Metcalfe, T. R. Birkhead, and P. F. Surai. 2003. Carotenoid modulation of immune function and sexual attractiveness in zebra finches. Science 300:125-127.

Bowmaker, J. K., L. A. Heath, S. E. Wilkie, and D. M. Hunt. 1997. Visual pigments and oil droplets from six classes of photoreceptor in the retinas of birds. Vision Research 37:2183-2194.

Burley, N., and C. B. Coopersmith. 1987. Bill color preferences of zebra finches. Ethology 76:133-151.

Burnham, K. P., and D. R. Anderson. 2002. Model selection and multimodel interference: a practical information-theoretic approach. Springer, New York.

Burnham, K. P., D. R. Anderson, and K. P. Huyvaert. 2011. AIC model selection and multimodel inference in behavioral ecology: some background, observations, and comparisons. Behavioral Ecology and Sociobiology 65:23-35.

Caves, E. M., P. A. Green, M. N. Zipple, D. Bharath, S. Peters, S. Johnsen, and S. Nowicki. 2020. Data from: Comparison of categorical color perception in two estrildid finches. American Naturalist, Dryad Digital Repository, https://doi.org/10.5061/dryad .stqjq2c1z.

Caves, E. M., P. A. Green, M. N. Zipple, S. Peters, S. Johnsen, and S. Nowicki. 2018. Categorical perception of colour signals in a songbird. Nature 560:365-367.
Caves, E. M., L. E. Schweikert, P. A. Green, M. N. Zipple, C. Taboada, S. Peters, S. Nowicki, et al. 2020. Variation in carotenoidcontaining retinal oil droplets correlates with variation in perception of carotenoid coloration. Behavioral Ecology and Sociobiology 74:93.

Cheney, K. L., N. F. Green, A. P. Vibert, M. Vorobyev, N. J. Marshall, D. C. Osorio, and J. A. Endler. 2019. An Ishihara-style test of animal colour vision. Journal of Experimental Biology 222:jeb189787.

Collins, S. A., C. Hubbard, and A. M. Houtman. 1994. Female mate choice in the zebra finch - the effect of male beak colour and male song. Behavioral Ecology and Sociobiology 35:21-25.

Colquitt, B. M., D. G. Mets, and M. S. Brainard. 2018. Draft genome assembly of the Bengalese finch, Lonchura striata domestica, a model for motor skill variability and learning. GigaScience $7: 1-6$.

Cronin, T. W., S. Johnsen, N. J. Marshall, and E. J. Warrant. 2014. Visual ecology. Princeton University Press, Princeton, NJ.

de Kogel, C. H., and H. J. Prijs. 1996. Effects of brood size manipulations on sexual attractiveness of offspring in the zebra finch. Animal Behaviour 51:699-708.

Eisner, E. 1960. The biology of the Bengalese finch. Auk 77:271-287.

Endler, J. A., and P. W. Mielke. 2005. Comparing entire colour patterns as birds see them. Biological Journal of the Linnean Society 86:405-431.

Green, P. A., N. C. Brandley, and S. Nowicki. 2020. Categorical perception in animal communication and decision-making. Behavioral Ecology 31:araa004.

Griffiths, R., M. C. Double, K. Orr, and R. J. G. Dawson. 1998. A DNA test to sex most birds. Molecular Ecology 7:1071-1075.

Harnad, S. R. 1987. Categorical perception: the groundwork of cognition. University of Cambridge Press, Cambridge.

Hart, N. S. 2001. The visual ecology of avian photoreceptors. Progress in Retinal and Eye Research 20:675-703.

Hart, N. S., J. C. Partridge, A. T. D. Bennett, and I. C. Cuthill. 2000. Visual pigments, cone oil droplets and ocular media in four species of estrildid finch. Journal of Comparative Physiology A 186:681-694.

Hempel de Ibarra, N., M. Giurfa, and M. Vorobyev. 2001. Discrimination of coloured patterns by honeybees through chromatic and achromatic cues. Journal of Comparative Physiology A 187:215-224.

Kuznetsova, A., P. B. Brockhoff, and R. H. B. Christensen. 2017. lmerTest package: tests in linear mixed effects models. Journal of Statistical Software 82, https://doi.org/10.18637/jss.v082.i13.

Liberman, A. M., K. S. Harris, H. S. Hoffman, and B. C. Griffith. 1957. The discrimination of speech sounds within and across phoneme boundaries. Journal of Experimental Psychology 54: 358-368.

Lind, O. 2016. Colour vision and background adaptation in a passerine bird, the zebra finch (Taeniopygia guttata). Royal Society Open Science 3:160383.

Osorio, D., and M. Vorobyev. 2005. Photoreceptor spectral sensitivities in terrestrial animals: adaptations for luminance and color vision. Proceedings of the Royal Society B 272:1745-1752.

Price, T. D. 2017. Sensory drive, color, and color vision. American Naturalist 190:157-170.

Price, T. D., M. C. Stoddard, S. K. Shevell, and N. I. Bloch. 2019. Understanding how neural responses contribute to the diversity of avian colour vision. Animal Behaviour 155:297-305. 
R Development Core Team. 2019. R: a language and environment for statistical computing. R Foundation for Statistical Computing, Vienna. https://www.R-project.org/.

Studdert-Kennedy, M., A. M. Liberman, K. S. Harris, and F. S. Cooper. 1970. Motor theory of speech perception: a reply to Lane's critical review. Psychological Review 77:234-249.

ten Cate, C., M. N. Verzijden, and E. Etman. 2006. Sexual imprinting can induce sexual preferences for exaggerated parental traits. Current Biology 76:1128-1132.

Vorobyev, M., and D. Osorio. 1998. Receptor noise as a determinant of colour thresholds. Proceedings of the Royal Society B 265:351-358.

Wehner, R. 1987. "Matched filters" — neural models of the external world. Journal of Comparative Physiology A 161:511-531.
Zipple, M. N., E. M. Caves, P. A. Green, S. Peters, S. Johnsen, and S. Nowicki. 2019. Categorical colour perception occurs in both signalling and non-signalling colour ranges in a songbird. Proceedings of the Royal Society B 286:0190524.

\section{References Cited Only in the Online Enhancements}

Olsson, U., and P. Alström. 2020. A comprehensive phylogeny and taxonomic evaluation of the waxbills (Aves: Estrildidae). Molecular Phylogenetics and Evolution 146:106757.

Associate Editor: J. Albert C. Uy Editor: Daniel I. Bolnick

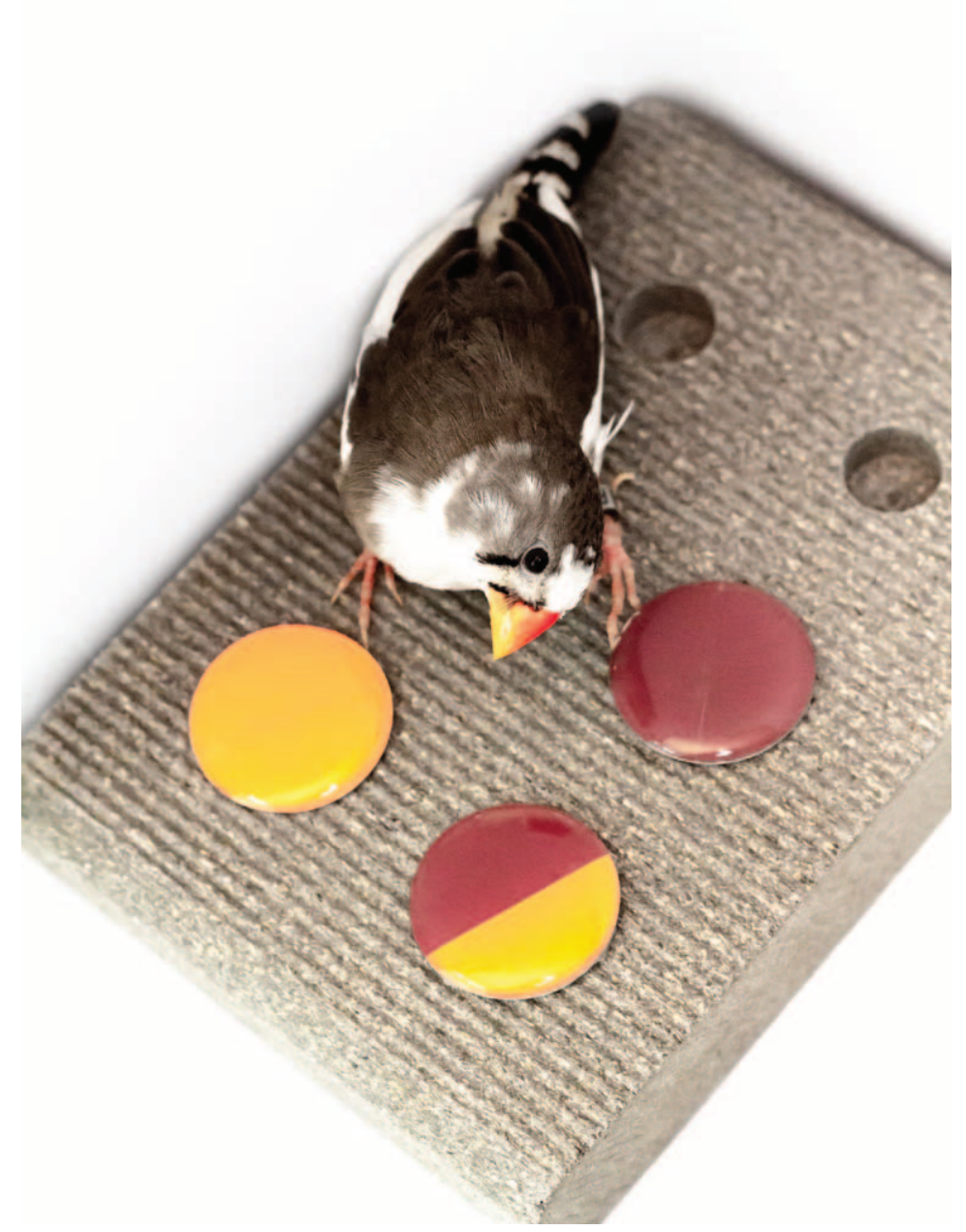

A female zebra finch Taeniopygia guttata engaged in the behavioral color discrimination task described in this article. Specifically, she has been trained to locate a food reward beneath disks comprising two colors. Photo: Ryan Huang, Terra Communications. 